\title{
A Condition for Positivity of Curvature
}

\author{
L. M. Chaves, A. Derdzinski and A. Rigas
}

\begin{abstract}
In this note we describe a necessary and sufficient condition, in order that a procedure of the type described in $[\mathbf{D}-\mathbf{R}$ ] yields metrics of positive sectional curvature in the total space of a principal bundle.
\end{abstract}

\section{Introduction}

The question of positivity of curvature on bundles arose in the middle 60's with $O$ 'Neills theorem [O], according to which riemannian submersions increase the sectional curvature.

In the early 70's Cheeger and Gromoll in their notable paper [C-G], showed that if an open manifold has a complete riemannian metric with non-negative sectional curvature then it is the total space of a vector bundle over a compact totally geodesic submanifold. Their result is actually quite stronger.

A natural question [Yau, problem no 6] is to ask whether the total spaces of bundles over compact manifolds with non-negative sectional curvature also admit complete metrics with the same property.

The approach to this question splits naturally in the cases of principal and vector bundles.

Although the vector bundle case can be treated independently [S-W], positive results follow from the principal bundle case through a simple application of O'Neill's theorem. See for example [G-M], [Ch], [R1], [R2].

This note, minus the application, was written in 1979 as an addendum to [D-R] and was never submitted for publication. Due to some revival of interest in the existence of metrics of non negative sectional curvature on vector bundles in recent years ([G], [W2], [S-W]) we thought its publication might be of some help.

We remark that the theorem was also known to L. Berard Bergery, at least in 
the case of $G=S^{1}$.

Strake and Walschap in [S-W] obtained a sufficient condition similar to (ii) for the non negativity of sectional curvatures in vector bundles.

\section{Main Result}

Let $P \stackrel{\pi}{\longrightarrow} G$ be a principal $G$-bundle, $G$ a compact Lie Group, over a compact manifold $M$ of dimension $n \geq 2$. Given a connection form $\omega$ in $P$, a metric $h$ in $M$, and a bi-invariant metric $\langle$,$\rangle in G$, one can define a family of metrics $g_{t}$, for $t>0$, in $P$ by

$$
g_{t}(X, Y)=h\left(\pi_{*}(X), \pi_{*}(Y)\right)+t\langle\omega(X), \omega(Y)\rangle
$$

(see $[\mathbf{J}]$ ), and ask when there are metrics of positive sectional curvature among the $g_{t}$.

With respect to each of these metrics, $G$ acts on $P$ by isometries, the fibers are totally geodesic and therefore by a theorem of Wallach [W1] we have that $G=S^{1}, G=S^{3}$ or $G=S O(3)$. Our objective here is to find a necessary and sufficient condition so that $\left(P, g_{t}\right)$ has positive sectional curvature for small enough $t$. This is natural since for small $t$ fibers we have "more curvature" and the curvature of the total space tends to increase.

Let $A d P=P \underset{A d}{\times \hat{G}}$ be the adjoint fiber bundle of $P$. The metric $\langle$,$\rangle of \hat{G}$ induces a metric in the fibers of $A d P$ that we will also denote by $\langle$,$\rangle . If \Omega$ is the curvature form of $\omega$ we can consider $\Omega$ as a form in $M$ with values in $A d P$.

Now, we can state our result.

Theorem. Let $P \stackrel{\pi}{\longrightarrow} M$ be a principal $G$-bundle with a connection $\omega$ over a compact manifold $M, \operatorname{dim} M \geq 2$, where $G=S^{3}, S O(3)$ or $S^{1}$. Fix a Riemannian metric $h$ in $M$ and a bi-invariant metric $\langle$,$\rangle in G$. Then the following conditions are equivalent:

i) There is a $t_{0}>0$, such that for $0<t \leq t_{0}\left(P, g_{t}\right)$ has positive sectional curvature.

ii) For any point $x$ in $M$, mutually orthonormal unit vectors $X, Y$ in $T_{x} M$ and any non-zero element $u$ of $A d(P)$ over $x$, we have

$$
\stackrel{h}{R}(X, Y, X, Y) \sum_{k=1}^{n}\left(\left\langle u, \Omega\left(X, X_{k}\right)\right\rangle\right)^{2}>\left(\left\langle u,\left(\nabla_{X} \Omega\right)(X, Y)\right\rangle\right)^{2}
$$

where $\stackrel{h}{R}$ is the curvature tensor of $(M, h), \Omega$ is the curvature form of $\omega, \nabla$ Bol. Soc. Bras. Mat., Vol. 23, Ns. 1-2, 1992 
is the covariant derivative of $A d(P)$ induced by $\omega$ and $\left\{X_{1}, \ldots, X_{n}\right\}$ is an arbitrary orthonormal basis of $T_{x} M$.

For the calculation of the components of the curvature tensor $R^{t}$ of $\left(P, g_{t}\right)$ we refer the reader to Jensen $[\mathbf{J}]$. The following convention for indices will be used:

$$
i \leq i, j, k, m \leq n=\operatorname{dim} M \text { and } n+1 \leq a, b, c, d, f \leq n+3 .
$$

Let $\left\{X_{n+1}, X_{n+2}, X_{n+3}\right\}$ be an orthonormal (o.n.) frame field of left invariant vector fields on $G$ with respect to $\langle$,$\rangle , i.e., an o.n. frame in the Lie algebra \hat{G}$ of $G$, taken to be $S^{3}$ or $S O(3)$ henceforth. Set $\left[X_{a}, X_{b}\right]=\sum_{c} C_{a b}^{c} X_{c}$. Since $\langle$,$\rangle is bi-invariant, the structure constants \left\{C_{a b}^{c}\right\}$ are skew-symmetric in every pair of indices. Let $\omega=\sum_{a} \omega^{a} X_{a}$ where the $\omega^{a}$ are l-forms on $P$ and observe that from the right action of $G$ on $P$, the vectors $\left\{X_{a}\right\}$ induce three fundamental vertical vector fields ([K-N p. 51]) $X_{n+1}^{*}, X_{n+2}^{*}, X_{n+3}^{*}$ on $P$, which are linearly independent at every point. Relative to the $g_{t}$-metric the fields

$$
\tilde{X}_{a} \equiv t^{-1 / 2} X_{a}^{*} \quad n+1 \leq a \leq n+3
$$

form an orthonormal vertical frame.

Let $\left\{X_{1}, \ldots, X_{n}\right\}$ be an $h-o . n$. frame field on an open $U \subseteq M$. We use the same notation for its lifting to a $g_{t}-o . n ., \omega$-horizontal frame field on $\pi^{-1}(U) \subseteq M$.

Define the following functions on $\pi^{-1}(U)$

$$
\begin{aligned}
& H_{i j}^{a}=\left\langle\Omega\left(X_{i}, X_{j}\right), X_{a}\right\rangle \\
& H_{i j ; k}^{a}=\left\langle\left(\nabla X_{k} \Omega\right)\left(X_{i}, X_{j}\right), X_{a}\right\rangle \\
& R_{i j k m}^{t}=g_{t}\left(R^{t}\left(X_{i}, X_{j}\right) X_{k}, X_{m}\right) \\
& R_{i j k a}^{t}=g_{t}\left(R^{t}\left(X_{i}, X_{j}\right) X_{k}, \tilde{X}_{a}\right), \text { etc. }
\end{aligned}
$$

We have

Proposition. ([J].)

1) $R_{i j k m}^{t}=R_{i j k m}^{t}=-t \sum_{a}\left(2 H_{i j}^{a} H_{k m}^{a}+H_{i k}^{a} H_{j m}^{a}-H_{i m}^{a} H_{j k}^{a}\right)$

2) $R_{i j m a}^{t}=-t^{1 / 2} H_{i j, m}^{\alpha}$

3) $R_{i j a b}^{t}=t \sum_{k}\left(H_{i k}^{a} H_{k j}^{b}-H_{i k}^{b} H_{k j}^{a}\right)-\sum_{c} H_{i j}^{c} C_{a b}^{c}$

4) $R_{i a j b}^{t}=-t \sum_{k} H_{i k}^{b} H_{k j}^{a}+\frac{1}{2} \sum_{c} H_{i j}^{c} C_{c b}^{a}$

5) $R_{a b i c}^{t}=0$

6) $R_{a b c d}^{t}=\frac{t^{-1}}{4} \sum_{f} C_{b f}^{a} C_{c d}^{f}$ 
All components of the curvature tensor can be obtained from these using the identities $R_{A B C D}=-R_{B A C D}=-R_{A B D C}, R_{A B C D}=R_{C D A B}$ and $R_{A B C D}+R_{A D B C}+R_{A C D B}=0$.

The following Lemma simplifies the calculation of sectional curvatures.

Lemma 1. Let $\sigma \subset T_{p} P$ be a bidimensional plane. There is always an orthonormal basis $\{X, Y\}$ of $\sigma$, relative to the metric $g_{1}$, such that

$$
\begin{cases}X=\alpha X_{i}+\beta X_{a} & \text { with } i \neq j, a \neq b \\ Y=\gamma X_{j}+\delta X_{b} & \alpha^{2}+\beta^{2}=\gamma^{2}+\delta^{2}=1\end{cases}
$$

Where $\left\{X_{i}, X_{j}\right\}$ is a $g_{1}$-orthonormal pair of horizontal vectors and $\left\{X_{a}, X_{b}\right\}$ a $g_{1}$-orthonormal pair of vertical vectors.

Proof. Let $\{X, Y\}$ be any $g_{1}$-orthonormal basis of $\sigma$ and $X=X^{H}+X^{V}$, $Y=Y^{H}+Y^{V}$ the decomposition into $\omega$-horizontal and vertical components. If $h\left(\pi_{*} X^{H}, \pi_{*} Y^{H}\right) \neq 0$ then the new $g_{1}$-orthonormal basis

$$
\tilde{X}=\cos (\theta) X-\sin (\theta) Y, \quad \tilde{Y}=\sin (\theta) X+\cos (\theta) Y
$$

with

$$
\operatorname{cotan}(2 \theta):=\frac{\left\|Y^{H}\right\|^{2}-\left\|X^{H}\right\|^{2}}{2 h\left(\pi_{*} X^{H}, \pi_{*} Y^{H}\right)}
$$

satisfies the condition.

Observe now that in relation to the metric $g_{t}\{X, Y\}$ is just an orthogonal basis and for $\tilde{X}_{a} \equiv t^{-1 / 2} X_{a}$, etc., we have

$$
\begin{aligned}
& \left\|\tilde{X}_{a}\right\|_{t}^{2}=g_{t}\left(\tilde{X}_{a}, \tilde{X}_{a}\right)=1, \\
& X=\alpha X_{i}+\beta t^{1 / 2} \tilde{X}_{a}, \\
& Y=\gamma X_{j}+\delta t^{1 / 2} \tilde{X}_{b}, \\
& \|X\|_{t}^{2}=\alpha^{2}+t \beta^{2} \\
& \|Y\|_{t}^{2}=\gamma^{2}+t \delta^{2} .
\end{aligned}
$$

For the sectional curvature $K_{t}(\sigma)$, relative to $g_{t}$, we have, using the above Lemma and Proposition:

$$
\begin{aligned}
K_{t}(\sigma)= & \frac{1}{\left(\alpha^{2}+\beta^{2} t\right)\left(\gamma^{2}+\delta^{2} t\right)}\left\{\alpha^{2} \gamma^{2} R_{i j i j}^{t}+2 t^{1 / 2} \alpha \beta \gamma^{2} R_{i j a j}^{t}\right. \\
& +2 t^{1 / 2} \alpha^{2} \gamma \delta R_{i j i b}^{t}+2 t \alpha \beta \gamma \delta R_{i b a j}^{t}+2 t \alpha \beta \gamma \delta R_{i j a b}^{t} \\
& +t \beta^{2} \gamma^{2} R_{j a j a}^{t}+2 t^{3 / 2} \beta^{2} \gamma \delta R_{a b a j}^{t}+2 t^{3 / 2} \alpha \beta \delta^{2} R_{i b a b}^{t}
\end{aligned}
$$




$$
\begin{aligned}
& \left.+t^{2} \beta^{2} \delta^{2} R_{a b a b}^{t}+t \alpha^{2} \delta^{2} R_{i b i b}^{t}\right\} \\
& =\frac{1}{\left(\alpha^{2}+\beta^{2} t\right)\left(\gamma^{2}+\delta^{2} t\right)}\left\{\alpha^{2} \gamma^{2}\left(R_{i j i j}^{h}-3 t \sum_{c}\left(H_{i j}^{c}\right)^{2}\right)\right. \\
& +2 \alpha \beta \gamma^{2} t H_{i j, j}^{a}-2 \alpha^{2} \gamma \delta t H_{i j, i}^{b} \\
& +2 \alpha \beta \gamma \delta t\left(t \sum_{k} H_{i k}^{a} H_{k j}^{b}-\frac{1}{2} \sum_{c} H_{i j}^{c} C_{c a}^{b}\right) \\
& \left.+2 \alpha \beta \gamma \delta t\left(t \sum_{k} H_{i k}^{a} H_{k j}^{b}-H_{i k}^{b} H_{k j}^{a}\right)-\sum_{c} H_{i j}^{c} C_{a b}^{c}\right) \\
& +\beta^{2} \gamma^{2} t\left(t \sum_{k}\left(H_{j k}^{a}\right)^{2}\right)+\beta^{2} \delta^{2} t^{2}\left(t^{-1} \frac{1}{4} \sum_{c}\left(C_{a b}^{c}\right)^{2}\right) \\
& \left.+\alpha^{2} \delta^{2} t\left(t \sum_{k}\left(H_{i k}^{b}\right)^{2}\right)\right\} .
\end{aligned}
$$

Let now $R_{t}(\sigma):=K_{t}(\sigma)\left(\alpha^{2}+\beta^{2} t\right)\left(\gamma^{2}+\delta^{2} t\right)$ and $K_{i j}^{M}$ be the sectional curvature in $M$ of the plane spanned by $\pi_{*} X_{i}$ and $\pi_{*} X_{j}$.

By the above formula, $R_{t}(\sigma)$ is the following binomial in the variable $t$ :

$$
\begin{aligned}
R_{t}(\sigma)= & K_{i j}^{M} \alpha^{2} \gamma^{2}+\left\{-3 \sum_{c}\left(H_{i j}^{c}\right)^{2} \alpha^{2} \gamma^{2}\right. \\
& +2 H_{i j, j}^{a} \alpha \beta \gamma^{2}-2 H_{i j, i}^{b} \alpha^{2} \gamma \delta \\
& \left.-3 \sum_{c} H_{i j}^{c} C_{a b}^{c} \alpha \beta \gamma \delta+\frac{1}{4} \sum_{c}\left(C_{a b}^{c}\right)^{2} \beta^{2} \delta^{2}\right\} t \\
& +\left\{2 \sum_{k}\left(2 H_{i k}^{a} H_{k j}^{b}-H_{i k}^{b} H_{k j}^{a}\right) \alpha \beta \gamma \delta\right. \\
& \left.+\sum_{k}\left(H_{j k}^{a}\right)^{2} \beta^{2} \gamma^{2}+\sum_{k}\left(H_{i k}^{b}\right)^{2} \alpha^{2} \delta^{2}\right\} t^{2}
\end{aligned}
$$

Now we need

Lemma 2. Given real parameters $A, B, C, D, E, F, G, K, L$ contained in a compact subset of $\mathbb{R}^{9}$, define the family of functions $\varphi_{t}: \mathbb{R}^{4} \rightarrow \mathbb{R}$, for $t>0$, by

$$
\begin{aligned}
\varphi_{t}(\alpha, \beta, \gamma, \delta):= & A \alpha^{2} \gamma^{2}+\left(B \alpha^{2} \gamma^{2}+C \alpha^{2} \gamma \delta+D \alpha \beta \gamma^{2}+E \alpha \beta \gamma \delta+\right. \\
& \left.+L \beta^{2} \delta^{2}\right) t+\left(K \alpha^{2} \delta^{2}+G \beta^{2} \gamma^{2}+F \alpha \beta \gamma \delta\right) t^{2}
\end{aligned}
$$

then the following conditions are equivalent.

(i) There exists a $t_{0}>0$, depending continuously on $A, B, \ldots, L$ such that

$$
\varphi_{t}(\alpha, \beta, \gamma, \delta)>0 \text { whenever } 0<t \leq t_{0}
$$


and

$$
\alpha^{2}+\beta^{2}=\gamma^{2}+\delta^{2}=1
$$

(ii) The parameters satisfy

$$
\begin{gathered}
A>0, G>0, K>0, \quad L>0 \\
4 A K>C^{2}, \quad 4 A G>D^{2} .
\end{gathered}
$$

Proof. Assume (i). Setting $\gamma=1$ together with $\alpha=1$ we get $A>0$ and $\alpha=1$ with $\delta=1$ implies $K>0$ and $\beta=1$ with $\gamma=1$ implies $L>0$ and $G>0$. If $\alpha=1, \beta=0$, writing (1) as a polynomial in $\gamma$ we have

$$
(A+t B) \gamma^{2}+t C \delta \gamma+t^{2} K \delta^{2}>0
$$

for $\gamma^{2}+\delta^{2}=1$ and small $t$.

Let $f: \mathbb{R}^{2} \rightarrow \mathbb{R}$ be defined by

$$
f(\gamma, \delta)=(A+t B) \gamma^{2}+t C \delta \gamma+t^{2} K \delta^{2}
$$

If $\delta=x \gamma$ then

$$
f(\gamma, x y)=\left(A+t B+t C x+t^{2} K x^{2}\right) \gamma^{2} .
$$

But, $f(\gamma, x \gamma)>0$ for $\gamma=\sqrt{\frac{1}{x^{2}+1}}$, and therefore $A+t B+t C x+t^{2} K x^{2}>0$ for all $x \in \mathbb{R}$, which implies that $4 A K>C^{2}$ for small $t$. Similarly, $\gamma=1, \delta=0$ implies $4 A G>D^{2}$.

Now assume (ii). As we saw above, $\varphi_{t}(1,0, \gamma, \delta)$ will be strictly positive for all small $t$, as a binomial in $\gamma$ with positive leading coefficient. Similarly $\varphi_{t}(\alpha, \beta, 1,0)>0$, so we may assume $\beta \delta \neq 0$. Divide $\varphi_{t}$ by $\beta^{2} \delta^{2}$, set $x=$ $\alpha \beta^{-1}, y=\gamma \delta^{-1}$ and obtain the following condition equivalent to the positivity of $\varphi_{t}$ :

$$
\begin{aligned}
& x^{2}\left[(A+t B) y^{2}+t C y+t^{2} K\right] \\
& \quad+x\left[t D y^{2}+t E y+t^{2} F y\right]+t L+t^{2} G y^{2}>0,
\end{aligned}
$$

for all real $x, y$ and all $t>0$ sufficiently small. The coefficient of $x^{2}$ is strictly positive for all real $y$ and all $t>0$ small enough as a binomial in $y$ with negative discriminant and positive leading coefficient. Condition (4) is then equivalent to the negativity of the corresponding discriminant, which is

$$
\left\{\begin{array}{l}
y^{2}\left[y^{2}\left(D^{2}-4 A G-4 t G B\right)+y(2 D(E+t F)-4 t C G)\right. \\
\left.+(E+t F)^{2}-4 t^{2} G K\right]<4 L\left[\left(\frac{1}{t} A+B\right) y^{2}+C y+t k\right]
\end{array}\right.
$$


The left hand side of (5) is a family of functions $\phi_{t}(y)$ in the variable $y$, depending on the small parameter $t>0$, satisfying

$$
\begin{aligned}
& \phi_{t}(0)=0 \\
& \left.\frac{d}{d y}\right|_{y=0} \phi_{t}(y)=0 \\
& \lim _{|y| \rightarrow \infty} \phi_{t}(y)=-\infty
\end{aligned}
$$

and uniformly bounded relative to the variables $t, y$ in a fixed neighborhood of $y=0$ as in Figure 1. Observe that for $t$ small enough $\phi_{t}$ is almost independent of $t$.

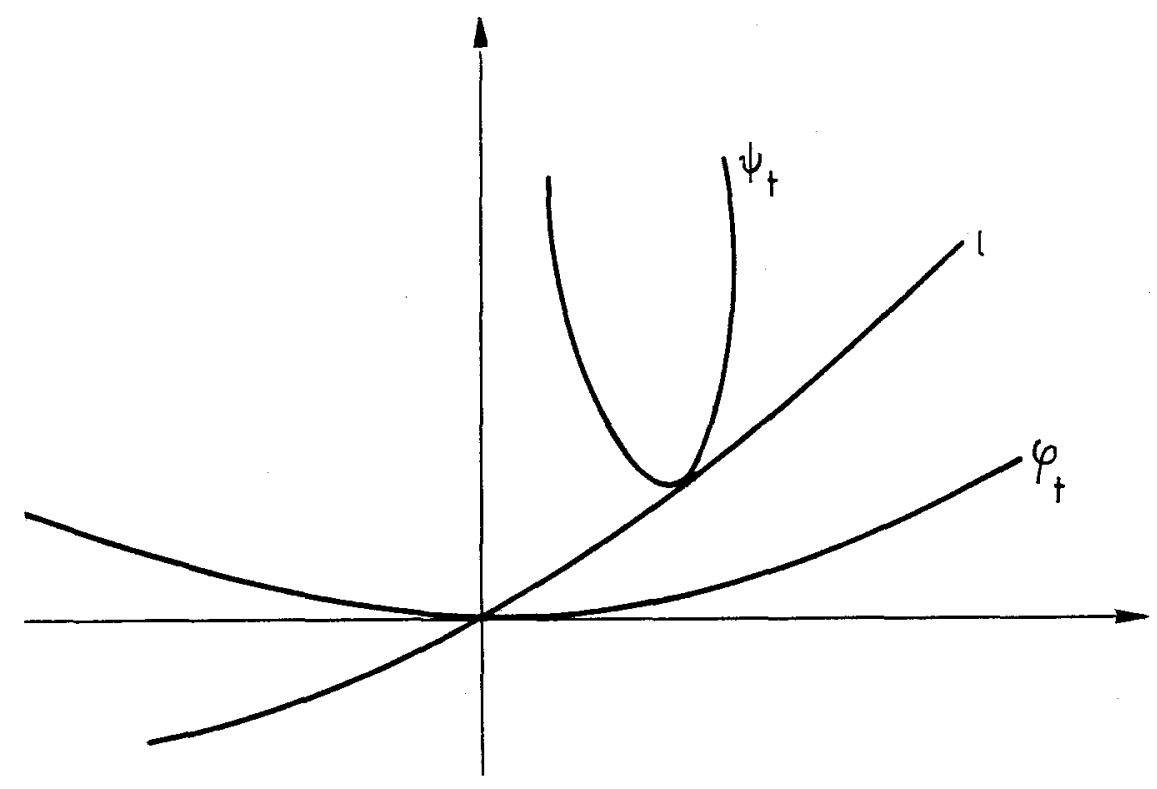

Figure 1

The right hand side of (5) is a family $\psi_{t}$ of binomials satisfying

$$
\lim _{t \rightarrow 0} \frac{d^{2}}{d y^{2}} \psi_{t}(y)=+\infty
$$

This means that each element of the family $\psi_{t}(y)$ becomes a thin parabola for small enough $t$, as in Figure 1.

The minimum of $\psi_{t}$, taken at $m_{t}=-\frac{t C}{2(A+t B)}$ is equal to

$$
\frac{L t}{A+t B}\left(4 A K-C^{2}+4 t B K\right)
$$


The curve of vertices $t \longmapsto l(t)=\left(m_{t}, \psi_{t}\left(m_{t}\right)\right)$ satisfies

$$
l(0)=(0,0) \text { and } l^{\prime}(0)=\left(-\frac{C}{2 A}, \frac{L}{A}\left(4 A K-C^{2}\right)\right)
$$

with second component positive in view of (ii). Therefore $l$ is not tangent to the horizontal axis at $(0,0)$ and the parabolas $\psi_{t}$ behave as in Figure 1.

Proof of Theorem. Let now in the formula for the sectional curvature

$$
\begin{array}{rlrl}
A & =K_{i j}^{M} & B & =-3 \sum_{c}\left(H_{i j}^{c}\right)^{2} \\
C & =-2 H_{i j, i}^{b} & D & =2 H_{i j, i}^{a} \\
E & =-\sum_{c} H_{i j}^{c} C_{a b}^{c} & L & =\frac{1}{4} \sum_{c}\left(C_{a b}^{c}\right)^{2} \\
K & =\sum_{k}\left(H_{i k}^{b}\right)^{2} & G & =\sum_{k}\left(H_{j k}^{a}\right)^{2} \\
F & =2 \sum_{k}\left(2 H_{j k}^{a} H_{k j}^{b}-H_{i j}^{b} H_{k j}^{a}\right) & \square
\end{array}
$$

By Lemma 2 the result follows.

Remark. The geometric meaning of condition (ii) is the positivity of all curvatures of the planes $\sigma$ generated by $\left\{X_{j}+s \tilde{X}_{a}, X_{j}\right\}, s \in \mathrm{R}$, since

$$
0<g_{t}\left(R^{t}\left(X_{i}+s X_{a}, X_{j}\right)\left(X_{i}+s X_{a}\right), X_{j}\right)=R_{i j i j}^{t}+2 R_{a j i j}^{t} s+R_{a j a j}^{t} s^{2}
$$

for all real $s$. Positivity of vertizontal curvatures $R_{a j a j}^{t}$ implies negativity of the discriminant

$$
\left(R_{a j i j}^{t}\right)^{2}<R_{i j i j}^{t} R_{a j a j}^{t}
$$

and by Jensen's Proposition one gets

$$
\left(H_{i j, j}^{a}\right)^{2}<R_{i j i j}^{M} \sum_{k}\left(H_{j k}^{a}\right)^{2} .
$$

Before applying the theorem to specific connections we recall the following facts from [W3].

a. If $S^{3} \cdots P \rightarrow M$ is a principal bundle with $K_{P}>0$ for some connection metric, then $\operatorname{dim} M=4 k$ and there is a 4 -form $\rho$ in $\wedge^{4}(M, \mathrm{R})$ with $\rho^{k} \neq 0$. This restricts the manifold $M$ and the first reasonable candidates are the $Q P^{n^{\prime}} s$ with $S^{4}$ leading the list.

b. Between all principal $S^{3}$ bundles over $S^{4}$ only the Hopf fibration $S^{3} \cdots S^{7} \rightarrow$ $S^{4}$ admits connections $\omega$ with associated $g_{t}$ of positive sectional curvature. 
c. It was proved by Chaves $[\mathrm{C}]$ that there is only a finite number of possible $S O(3)$-fat $S O(4)$ principal bundles over any compact orientable 4-dimensional manifold. He also showed that over $C P^{2}$ there is just one such that reduces to the bundle $U(2) \ldots S U(3) \rightarrow \mathbb{C} P^{2}$. This gives rise to infinitely many topologically distinct associated 3-sphere bundles over $C P^{2}$ with positive sectional curvature. These examples belong to the well known seven dimensional Wallach examples [A-W].

\section{Application to the Geometry of Instantons}

Our application amounts to checking which instantons of the Hopf bundle induce metrics of positive sectional curvature on $S^{7}$.

Let $S^{3} \cdots S^{7} \rightarrow S^{4}$ be the Hopf bundle. In [A-H-S] is shown that the moduli space of irreducible self dual connections of the Hopf bundle is a five dimensional manifold, diffeomorphic to the disk $D^{5}$, given by a 5 -parameter family of instantons obtained through the basic instanton [A, p.23]. By the natural symmetries [ $\mathbf{L}, \mathbf{p . 4 6}]$ we can consider simply a 1-parameter family given by the following construction: Working with the tautological vector bundle over $S^{4}$ and taking a trivialization given by stereographic projection, the family of instantons is

$$
A^{\lambda}=\operatorname{Im}\left(\frac{x d \bar{x}}{\lambda^{2}+|x|^{2}}\right)
$$

with field

$$
R^{\lambda}=\frac{\lambda^{2}}{\left(\lambda^{2}+|x|^{2}\right)^{2}} d x \wedge d \bar{x}
$$

where $0<\lambda \leq 1$ and $\operatorname{Im}$ is the imaginary part. For the relations between the curvature form in $S^{\mathbf{7}}$ and $R^{\lambda}$ see [F-U, p.102].

Let $e_{1}=(1,0,0,0), \ldots, e_{4}=(0,0,0,1)$ be the canonical fields in $\mathbb{R}^{4}$ and

$$
X_{1}=\left(\frac{1+|x|^{2}}{2}\right) e_{1}, \ldots, X_{4}=\left(\frac{1+|x|^{2}}{2}\right) e_{4}
$$

an orthonormal frame in $\mathrm{R}^{4}$ in relation to the stereographic metric. If $V_{1}=i$, $V_{2}=j$ and $V_{3}=k$ are the canonical basis of the algebra $\operatorname{Im} \not H$, where $\mathcal{H}$ denotes the quaternions, then

$$
\begin{aligned}
H_{i j}^{a} & =\left\langle R^{\lambda}\left(X_{i}, X_{j}\right), V_{a}\right\rangle \\
H_{i j, i}^{a} & =\left\langle\left(\nabla_{X_{i}} R^{\lambda}\right)\left(X_{i}, X_{j}\right), V_{a}\right\rangle
\end{aligned}
$$


(Note that $\nabla R^{\lambda}=\left[\nabla, R^{\lambda}\right][\mathbf{L}, \mathbf{p . 1 9}]$ )

$$
R^{\lambda}\left(X_{i}, X_{j}\right)=\frac{\lambda^{2}\left(1+|x|^{2}\right)^{2}}{4\left(\lambda^{2}+|x|^{2}\right)^{2}} d x \wedge d \bar{x}\left(e_{i}, e_{j}\right)
$$

$\left(\nabla_{X_{i}} R^{\lambda}\right)\left(X_{i}, X_{j}\right)=\nabla_{X_{i}}\left(R^{\lambda}\left(X_{i}, X_{j}\right)\right)-R^{\lambda}\left(\tilde{\nabla}_{X_{i}} X_{i}, X_{j}\right)-R^{\lambda}\left(X_{i}, \tilde{\nabla}_{X_{i}} X_{j}\right)$ where $\tilde{\nabla}$ is the Levi-Civita connection of the stereographic metric.

We have $\tilde{\nabla}_{X} Y=D_{X} Y+X(\varphi) Y+Y(\varphi) X-\langle X, Y\rangle \operatorname{grad} \varphi$ where $D$ is the Levi-Civita connection of the flat metric in $\mathrm{R}^{4}, \varphi(x)=\ln (2)-\ln \left(|x|^{2}+1\right)$ and $\operatorname{grad} \varphi$ is the gradient field of $\varphi[\mathbf{P}, \mathbf{p} .178]$. Then, by a straightforward calculation we get

$$
\begin{aligned}
R^{\lambda}\left(\tilde{\nabla}_{X_{i}} X_{i}, X_{j}\right)= & \frac{\lambda^{2}}{4}\left(\frac{1+|x|^{2}}{\lambda^{2}+|x|^{2}}\right)^{2}\left\{-x_{i} d x \wedge d \bar{x}\left(e_{i}, e_{j}\right)+d x \wedge d \bar{x}\left(x, e_{j}\right)\right\} \\
R^{\lambda}\left(X_{i}, \tilde{\nabla}_{X_{i}} X_{j}\right)=0 & \\
\nabla_{X_{i}}\left(R^{\lambda}\left(X_{i}, X_{j}\right)\right)= & \frac{1+|x|^{2}}{2} \nabla_{e_{i}}\left(\frac{\lambda^{2}}{4}\left(\frac{1+|x|^{2}}{\lambda^{2}+|x|^{2}}\right)^{2} d x \wedge d \bar{x}\left(e_{i}, e_{j}\right)\right) \\
= & \frac{1+|x|^{2}}{2}\left\{e_{i}\left(\frac{\lambda^{2}}{4}\left(\frac{1+|x|^{2}}{\lambda^{2}+|x|^{2}}\right)^{2}\right) d x \wedge d \bar{x}\left(e_{i}, e_{j}\right)\right. \\
& \left.+\left[A\left(e_{i}\right), \frac{\lambda^{2}}{4}\left(\frac{1+|x|^{2}}{\lambda^{2}+|x|^{2}}\right)^{2} d x \wedge d \bar{x}\left(e_{i}, e_{j}\right)\right]\right\} .
\end{aligned}
$$

A tedious calculation yields

$$
\begin{aligned}
\nabla_{X_{i}}\left(R^{\lambda}\left(X_{i}, X_{j}\right)\right)= & \lambda^{2}\left(\frac{1+|x|^{2}}{\lambda^{2}+|x|^{2}}\right)^{2}\left\{\frac{1}{2}\left(\frac{\lambda^{2}-1}{\lambda^{2}+|x|^{2}}\right) x_{i} d x \wedge d \bar{x}\left(e_{i}, e_{j}\right)\right. \\
& \left.+\frac{1+|x|^{2}}{8\left(\lambda^{2}+|x|^{2}\right)}\left[\operatorname{Im}\left(x d \bar{x}\left(e_{i}\right)\right), d x \wedge d \bar{x}\left(e_{i}, e_{j}\right)\right]\right\}
\end{aligned}
$$

Collecting terms now we get $\left.\nabla_{X_{i}} R^{\lambda}\right)\left(X_{i}, X_{j}\right)$.

In the particular case $i=1, j=2$,

$$
\begin{aligned}
\left.\nabla_{X_{1}} R^{\lambda}\right)\left(X_{1}, X_{2}\right)= & \lambda^{2}\left(\frac{1+|x|^{2}}{\lambda^{2}+|x|^{2}}\right)^{2}\left\{\frac{1-\lambda^{2}}{\lambda^{2}+|x|^{2}} x_{1} V_{1}+\right. \\
& \left.+\frac{1-\lambda^{2}}{2\left(\lambda^{2}+|x|^{2}\right)} x_{3} V_{3}+\frac{\lambda^{2}-1}{2\left(\lambda^{2}+|x|^{2}\right)} x_{4} V_{2}\right\}
\end{aligned}
$$


and therefore

$$
\begin{aligned}
& H_{12,1}^{1}=\lambda^{2}\left(1-\lambda^{2}\right)\left(1+|x|^{2}\right)^{2}\left(\lambda^{2}+|x|^{2}\right)^{-3} x_{1} \\
& H_{12,1}^{2}=\frac{\lambda^{2}}{2}\left(\lambda^{2}-1\right)\left(1+|x|^{2}\right)^{2}\left(\lambda^{2}+|x|^{2}\right)^{-3} x_{4} \\
& H_{12,1}^{3}=\frac{\lambda^{2}}{2}\left(1-\lambda^{2}\right)\left(1+|x|^{2}\right)^{2}\left(\lambda^{2}+|x|^{2}\right)^{-3} x_{3}
\end{aligned}
$$

We also have

$$
H_{1 s}^{1}=\frac{\lambda^{2}}{4}\left(\frac{1+|x|^{2}}{\lambda^{2}+|x|^{2}}\right)^{2}\left\langle d x \wedge d \bar{x}\left(e_{1}, e_{s}\right), V_{1}\right\rangle
$$

and therefore

$$
H_{1 s}^{1}=-\frac{\lambda^{2}}{4}\left(\frac{1+|x|^{2}}{\lambda^{2}+|x|^{2}}\right)^{2}
$$

if $s=2$, and zero if $s \neq 2$.

Now we test the validity of

$$
\left(H_{12,1}^{1}\right)^{2}<\sum_{s=1}^{4}\left(H_{1 s}^{1}\right)^{2}
$$

that is

$$
\left(\frac{1-\lambda^{2}}{\lambda^{2}+|x|^{2}}\right)^{2}\left(x_{1}\right)^{2}<\frac{1}{4}
$$

which is equivalent to

$$
f(x)=\left(1-\lambda^{2}\right)^{2}\left(\frac{x_{1}}{\lambda^{2}+|x|^{2}}\right)^{2}-\frac{1}{4}<0 .
$$

But, $|x|^{2} \geq\left(x_{1}\right)^{2}$ and, therefore, if

$$
g\left(x_{1}\right)=\left(1-\lambda^{2}\right)^{2}\left(\frac{x_{1}}{\lambda^{2}+x_{1}^{2}}\right)^{2}-\frac{1}{4} .
$$

we have $g\left(x_{1}\right) \geq f(x)$ (where $x=\left(x_{1}, x_{2}, x_{3}, x_{4}\right)$ ). Since

$$
\frac{d}{d x_{1}} g\left(x_{1}\right)=2\left(1-\lambda^{2}\right)^{2} \frac{x_{1}}{\lambda^{2}+x_{1}^{2}}\left(\lambda^{2}-x_{1}^{2}\right),
$$

the critical points of $g$ are $x_{1}=0, x_{1}=\lambda$, and $x_{1}=-\lambda$. Then we have

$$
\begin{aligned}
& g(0)=-\frac{1}{4} \\
& g(\lambda)=g(-\lambda)=\frac{\left(1-\lambda^{2}\right)^{2}}{4 \lambda^{2}}-\frac{1}{4},
\end{aligned}
$$


and

$$
\lim _{x_{1} \rightarrow \pm \infty} g\left(x_{1}\right)=-\frac{1}{4} .
$$

Therefore (6) is true if and only if $g(\lambda)<0$, i.e., $\lambda>\frac{-1+\sqrt{5}}{2}$. By symmetry we get the same inequality for all $i, j$ and therefore by the theorem we have:

Corollary. The instantons defined by $A^{\lambda}$, such that $\left(S^{7}, g_{t}\right)$ has positive sectional curvature for all small enough $t$ are the ones with $\lambda$ in $\left(\frac{-1+\sqrt{5}}{2}, 1\right]$.

Remark. It is obvious that to check condition (ii) of the theorem can lead to a quite cumbersome calculation even in the simplest of cases. It is hoped that an easier to use version can be found.

\section{References}

[A] M. F. Atiyah "The geometry of Yang-Mills fields" Scuola Normale Superiore Pisa, (1979).

[A-H-S] M. F. Atiyah, N. Hitchin and I. Singer "Self-duality in four dimensional Riemannian geometry" Proc. Roy. Soc. London Ser A 362 (1978) 425-461.

[B-L] J. -P. Bourguignon and H. B. Lawson "Stability and isolation phenomena for Yang-Mills fields" Commun. Math. Phys. (1981) 189-230.

[C] L. M. Chaves "A theorem of finiteness for fat bundles" (Preprint) Unicamp (1992).

[Ch] J. Cheeger "Some examples of manifolds of non-negative curvature", J. Diff. Geom. 8(1974) 623-628.

[C-G] J. Cheeger and D. Gromoll "On the structure of complete open manifolds of non-negative curvature" Ann. of Math. 96 (1972) 413-443.

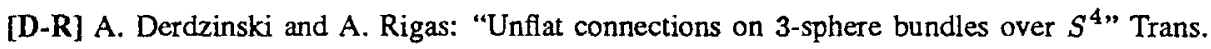
AMS v. $265 n^{\circ} 2$ (1981) $485-493$.

[F-U] D. Freed and K. Uhlenbeck "Instantons and four - manifolds" MRSI publications, v. 1, Springer-Verlag (1984).

[G] M. A. Bozola Grou: "Curvature on fibre bundles" (in Portuguese) Ph. D. Thesis, Unicamp 1987.

[G-M] D. Gromoll and W. Meyer "An exotic sphere of non-negative sectional curvature" Ann. of Math. 100 (1974) 407-411.

[J] G. R. Jensen "Einstein metrics on principal fibre bundles" J. Diff. Geom. 8 (1973) 599-614.

[L] H. B. Lawson "The theory of gauge fields in four dimensions" AMS, Regional conference series in Math, $\mathrm{n}^{\circ} 58$ (1985).

[O] B. O'Neill "The fundamental equations of a submersion" Michigan Math. I. 13 (1966) 459-469. 
[P] W. A. Poor "Differential Geometric structures" Mac Graw-Hill, (1981).

[R1] A. Rigas "Geodesic spheres as generators of the homotopy groups of $O, B O$ " J. Diff. Geom., 13 (1978) 527-545.

[R2] A. Rigas "Some bundles of non-negative curvature" Math. Ann. 232 (1978) 187-193.

[S-W] M. Strake and G. Walschap: "Connection metrics of non negative curvature on vector bundles" preprint, UCLA 1989.

[W1] N. R. Wallach: "Compact homogeneous Riemannian manifolds with strictly positive curvature" Ann. of Math. 96 (1972) 277-295.

[W2] G. Walschap "Nonnegatively curved manifolds with souls of codimension 2" J. Diff. Geom. 27(1988) 525-537.

[W3] A. Weistein "Fat bundles and sympletic manifolds" Adv. in Math. 37 № 3 (1980) 239-250.

[Y] S-T. Yau "Seminar on Differential Geometry" Ann. of Math. Studies, no 102, Princeton Univ. Press (1982).

L. M. Chaves

ESAL

37.200-000, Lavras, MG

Brazil

A. Derdzinski

Department of Mathematics

Ohio State University

Columbus, $\mathrm{OH} 43210$

USA

A. Rigas

IMECC - Unicamp

P. O. Box 6065

13081, Campinas, SP

Brazil 